NOTICE: this is the author's version of a work that was accepted for publication in Performance Evaluation. Changes resulting from the publishing process, such as peer review, editing, corrections, structural formatting, and other quality control mechanisms may not be reflected in this document. Changes may have been made to this work since it was submitted for publication. A definitive version was subsequently published in Performance Evaluation, vol. 66, issues 3-5, March 2009, pp. 158-172, doi:10.1016/j.peva.2008.10.003.

\title{
On the Optimality of Field-Line Routing in Massively Dense Wireless Multi-Hop Networks
}

\author{
Esa Hyytiäa ${ }^{\mathrm{a}}$ Jorma Virtamo ${ }^{\mathrm{b}}$ \\ ${ }^{a}$ Telecommunications Research Center Vienna, Donau-City Straße 1, 1220 Vienna, Austria \\ b Department of Communications and Networking, Helsinki University of Technology, Finland
}

\section{A B S T R A C T}

We consider the load balancing problem in large wireless multi-hop networks by applying the continuum approximation. The task is to find routes, geometric curves, such that the maximal traffic load in the network is minimized. In finite fixed networks, multi-path routes generally yield a lower congestion and thus allow higher throughput. In contrast, we show that in dense wireless multi-hop networks, the optimal load balancing can be achieved by a destination based single-path routing referred to as field-line routing. This is because any routing can be transformed to the corresponding field-line routing with the same or better performance, by using as paths, the field lines of the so-called destination flow associated with the original routing. The concepts are illustrated with two examples. In the case of a unit disk with unit traffic, the maximal load of 0.389 of a multi-path routing system is reduced to 0.343 by using the field-line routing. Similar improvements are also demonstrated for the unit square.

Keywords: Wireless multi-hop network, Multi-path, Single-path, Load balancing.

\section{Introduction}

The load balancing problem in fixed networks is a well-known problem for which several formulations have been proposed. Typically, one is asked to find such routes that minimize the maximal link load. In general, lower congestion can be obtained by dividing the traffic flows into several routes, i.e., by using multi-path routes. Frequently the problem description lends itself to an efficient formulation as an LPproblem - see, for example, [1]. The approach is generic and can be applied to different kinds of networks ranging from packet switching to lightpath routed optical networks.

In this paper, we consider the load balancing problem in a wireless multi-hop network in the setting of a so-called massively dense multi-hop network. A massively dense multi-hop network means that 1) there are nodes practically everywhere, and 2) that a typical path between two nodes consists of a large number of hops, (i.e., the transmission range is several orders smaller in magnitude than the diameter of the network). Note that in the considered type of networks, the traffic load consists almost solely of the relay traffic. At the limit, there is a strong separation between the microscopic level, corresponding to the immediate neighborhood of a given node, and the macroscopic level, corresponding to the end-to-end connections. At the microscopic level, the nodes are simply concerned about forwarding a given packet to the direction 
defined by the chosen routing. In practice, this means that, locally, a packet is forwarded to the furthest reachable node in that direction. ${ }^{1}$ At the macroscopic level, one is concerned with the end-to-end paths, and the assumption of a strong separation between the different scales justifies describing the underlying network as a continuous medium and the paths as smooth continuous curves [2-10]. In the present paper, we focus on studying the optimal paths at the macroscopic level.

The exact load balancing problem in dense wireless multi-hop networks considered in the present paper was first formulated in [11] and has also been studied in [10,12]. In particular, in [11,12] we focused on single-path routing with curvilinear paths and noted that multiple sets of single-path routes can straightforwardly be combined to multi-path routing by randomly choosing one of the single-path routes for each packet. In the present paper, we show that, with respect to the load balancing problem, the optimal solution can always be achieved by so-called field-line routing, which consists of destination based single-path routes constructed in a special way. This new result is in striking contrast to finite fixed networks, where the restriction to single-path routes can severely limit the performance of the network. The optimality of field-line (single-path) routes is a consequence of another result of this paper, which states that, starting from any routing system, the scalar flux will decrease or remain the same everywhere if the routing system is replaced with the corresponding field-line routing. This procedure is demonstrated by examples, where we are indeed able to reduce the maximal scalar flux by using the field-line routing. Furthermore, we show that the optimal paths are unique and bidirectional in the so-called bottleneck region, where each optimal solution achieves the maximum of the scalar packet flux.

The rest of the paper is organized as follows. In Section 1.1 the related work is reviewed. Then, in Section 2 we introduce the basic concepts and define the load balancing problem. Our main results are presented in Section 3, where we prove that the optimal solution can be achieved by the destination based single-path routes. The uniqueness and bidirectionality of the paths in the so-called bottleneck area are also established. In Section 4, the framework and the new results are illustrated by means of examples, and Section 5 contains the conclusions.

\section{$1.1 \quad$ Related Work}

The research community has recently shown considerable interest in different routing problems in dense wireless multi-hop networks. The idea of modeling routes as continuous curves at the macroscopic level was introduced in [3] and [4]. However, the trajectory based forwarding (TBF) scheme, proposed by Niculescu and Nath [13], already provides the connection between the macroscopic and microscopic levels. Another closely related concept is the so-called geometric routing (or geographic routing) paradigm [14-16], where each packet carries the information (directly or indirectly) about the location of the destination node. Typically the nodes along the path simply forward the packet in a greedy manner to the neighbor which is closest to the destination. In the same fashion, Baumann et al., in [17], propose a robust and scalable anycast routing scheme for large wireless mesh networks based on temperature fields. The scheme, referred to as HEAT, assigns each node a temperature in such a way that the temperature always increases towards the gateway node(s).

The shortest path routes tend to concentrate traffic to the center of the network. This is particularly true of

\footnotetext{
1 To be more precise, the task is to find a coordinated forwarding scheme that maximizes the sustainable packet flow in a given direction. Finding the maximum is an interesting and challenging problem but is outside the scope of this paper. The sustainable maximum can be shared between different directions in a time-sharing manner and sets an upper bound to the scalar flux (see Def. 4). This allows us to assimilate the scalar flux with the local traffic load.
} 
uniform traffic demands [8,11, 18]. In [18], Pham and Perreau, and later, in [19], Ganjali and Keshavarzian have studied the possible gain from using $k$-shortest paths instead of one. By using approximative modeling techniques, Pham et al. argue that the use of multi-path routing always results in improvement to throughput. However, the results by Ganjali et al., based on modeling the $k$ shortest paths as a rectangle between the node pairs, suggest that this is not always the case, unless a huge number of multiple paths are allowed for each pair of nodes. This is due to the fact that, in the limit of massively dense networks, any finite number of shortest paths reduces to a single line segment between the given nodes.

A considerable amount of recent work relies on strong analogy with physical systems. In [20] a set of paths are derived, based on the heat conduction in a unit disk. Similarly, Kalantari and Shayman [3] have studied dense wireless multi-hop networks by leaning to the theory of electrostatics. In particular, the authors consider a routing problem where a large number of nodes are sending data to a single destination. In this case the optimal paths are obtained by solving a set of partial differential equations similar to Maxwell's equations. Using a similar analogy with electrostatics, Toumpis and Tassiulas [2] consider a related problem of optimal placement of the nodes in a dense sensor network. It is assumed that, at any point in the network, the information flows exactly in one direction.

Another interesting analogy is provided by geometrical optics. Catanuto et al. [6,7] have optimized the routes by exploiting this analogy. The communication cost, which is related to the index of refraction in the analogy, is defined as a function of the node density. In [10], Popa et al. study the load balancing problem using the linear programming approach and are able to show that the optimal paths can be expressed as shortest (minimum cost) paths with a certain metric. This means that the geometrical optics analogy is accurate, with the index of refraction defining the metric. The result provides an alternative approach to some of the results in the current paper, first presented in [20]. The main obstacle with the approach seems to be the technical difficulties in actually determining the metric and computing the traffic load and, as a practical solution, the authors propose a heuristic set of paths referred to as curveball routing.

Finally, we note that a comprehensive survey on different modeling approaches and problem formulations has been given by Toumpis [5,9].

\section{Preliminaries and Definitions}

To start with, we first need to define the traffic that is offered to the network located in some area denoted by $\mathcal{A}$. In our setting, the nodes form a continuum and thus it is convenient to define the traffic demands as densities:

Definition 1 (traffic demand density) The rate of flow of packets from a differential area element $d A$ about $\mathbf{x}_{1}$ to a differential area element $d A$ about $\mathbf{x}_{2}$ is $\lambda\left(\mathbf{x}_{1}, \mathbf{x}_{2}\right) \cdot d A^{2}$, where $\lambda\left(\mathbf{x}_{1}, \mathbf{x}_{2}\right)$ is called the traffic demand density [pkts/s/m $\left.\mathrm{m}^{4}\right]$.

These end-to-end traffic demands are then satisfied by forwarding each packet from the source $\mathbf{x}_{1}$ to the destination $\mathbf{x}_{2}$ over a multi-hop route, which, in our context, translates to a continuous path from $\mathrm{x}_{1}$ to $\mathbf{x}_{2}$. The set of paths is denoted by $\mathcal{P}$. In this set there is at least one path for every source-destination pair $\left(\mathbf{x}_{1}, \mathbf{x}_{2}\right)$.

From the point of view of the forwarding load (scalar flux, see Def. 4), the direction of the traffic is immaterial. It follows that, without a loss of generality, one can assume symmetric traffic demands:

Definition 2 (symmetric traffic demands) The traffic demands are said to be symmetric if

$$
\lambda\left(\mathbf{x}_{1}, \mathbf{x}_{2}\right)=\lambda\left(\mathbf{x}_{2}, \mathbf{x}_{1}\right), \quad \forall \mathbf{x}_{1}, \mathbf{x}_{2} \in \mathcal{A}
$$




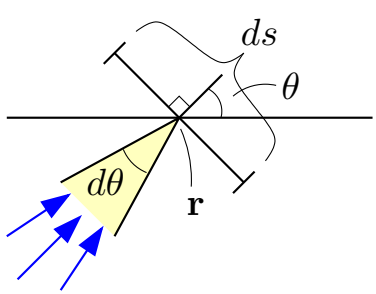

a) angular flux $\varphi(\mathbf{r}, \theta)$

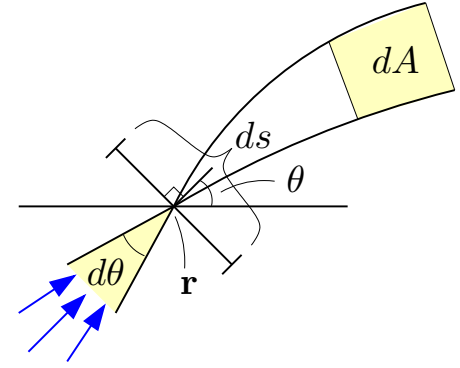

b) angular d-flow $\varphi(\mathbf{r}, \theta ; \mathbf{x})$

Fig. 1. Angular flux $\varphi(\mathbf{r}, \theta)$ is the rate of packets crossing a small line segment $d s$ in angle $(\theta, \theta+d \theta)$ divided by $d \phi \cdot d s$ in the limit $d \theta, d s \rightarrow 0$. Angular d-flow density is equal to the angular flux of packets having a destination in $d A$ about $\mathbf{x}$ in the limit $d A, d \theta, d s \rightarrow 0$.

As part of our earlier work, in [11,12], we have defined the load balancing problem in dense multi-hop networks as a minmax problem for the scalar packet flux. Scalar packet flux, in turn, is defined in terms of so-called angular packet flux (see Fig. 1):

Definition 3 (angular flux) Angular flux of packets at $\mathbf{r}$ in direction $\theta$, denoted by $\varphi(\mathbf{r}, \theta)$, is equal to the rate $[1 / \mathrm{s} / \mathrm{m} / \mathrm{rad}]$ at which packets flow in the angle interval $(\theta, \theta+d \theta)$ across a small line segment of the length $d s$ perpendicular to direction $\theta$ at point $\mathbf{r}$ divided by $d s \cdot d \theta$ in the limit when $d s \rightarrow 0$ and $d \theta \rightarrow 0$.

Definition 4 (scalar flux) Scalar flux of packets [1/s/m] at $\mathrm{x}$ is given by

$$
\Phi(\mathbf{r})=\Phi(\mathcal{P}, \mathbf{r})=\int_{0}^{2 \pi} \varphi(\mathcal{P}, \mathbf{r}, \theta) d \theta .
$$

We note that these packet fluxes are defined in analogy with the corresponding particle fluxes in physics, e.g., in neutron transport theory [21]. The load balancing problem in the context of massively dense multihop networks is as follows:

Definition 5 (load balancing problem) Find the set of paths $\mathcal{P}$ minimizing the maximal scalar flux,

$$
\underset{\mathcal{P}}{\arg \min } \max _{\mathbf{r}} \Phi(\mathcal{P}, \mathbf{r}) .
$$

For the load balancing problem, two useful lower bounds are given in [11]:

\section{Proposition 6 (distance bound)}

$$
\max _{\mathbf{r}} \Phi(\mathcal{P}, \mathbf{r}) \geq \frac{\Lambda \cdot \bar{\ell}}{A}
$$

where $\bar{\ell}=\bar{\ell}\left(\mathcal{P}, \lambda\left(\mathbf{x}_{1}, \mathbf{x}_{2}\right)\right)$ denotes the mean path length.

Proposition 7 (cut bound) For any curve $\mathcal{C}$ separating the domain $\mathcal{A}$ into two disjoint subdomains $\mathcal{A}_{1}$ and $\mathcal{A}_{2}$, it holds that

$$
\Phi_{\mathrm{opt}} \geq \frac{1}{L} \int_{\mathcal{A}_{1}} d^{2} \mathbf{x}_{1} \int_{\mathcal{A}_{2}} d^{2} \mathbf{x}_{2}\left(\lambda\left(\mathbf{x}_{1}, \mathbf{x}_{2}\right)+\lambda\left(\mathbf{x}_{2}, \mathbf{x}_{1}\right)\right),
$$

where $L$ is the length of the curve $\mathcal{C}$ and the double integral gives the rate of packets between $\mathcal{A}_{1}$ and $\mathcal{A}_{2}$ (both directions included).

The first inequality follows from the fact that the maximal scalar flux cannot be less than the mean scalar flux, which is equal to the average progress density in the given area. The second inequality is obtained 
by considering the average scalar flux along the cut. The distance bound (4) is further lower bounded by $\Lambda \cdot \bar{\ell}_{\mathrm{SP}} / A$, where SP refers to shortest paths, yielding a strict lower bound for all path sets.

For our developments, it is useful to single out flows associated with the traffic with a given destination $\mathrm{x} \in \mathcal{A}$. In the following definitions the prefix $\mathrm{d}$ stands for destination.

Definition 8 (angular d-flux density) Angular d-flux density, denoted by $\varphi(\mathbf{r}, \theta ; \mathbf{x})\left[1 / \mathrm{s} / \mathrm{m}^{3} / \mathrm{rad}\right]$, is equal to the angular flux $\varphi(\mathbf{r}, \theta)$ divided by $d A$ resulting from the packets having their final destination in small area $d A$ about $\mathbf{x}$, in the limit when $d A \rightarrow 0$.

The concept of angular d-flux density is illustrated in Fig. 1 b). By definition,

$$
\varphi(\mathbf{r}, \theta)=\int_{\mathcal{A}} \varphi(\mathbf{r}, \theta ; \mathbf{x}) d^{2} \mathbf{x} .
$$

Definition 9 (d-flow intensity) Destination flow intensity, or d-flow intensity for short, of packets at $\mathbf{r}$ having destination $\mathbf{x}$, denoted by $\mathbf{J}(\mathbf{r}, \mathbf{x})\left[1 / \mathrm{s} / \mathrm{m}^{3}\right]$, is equal to

$$
\mathbf{J}(\mathbf{r}, \mathbf{x})=\int_{0}^{2 \pi} \varphi(\mathbf{r}, \theta ; \mathbf{x}) \mathbf{e}_{\theta} d \theta
$$

where $\mathbf{e}_{\theta}$ is the unit vector in direction $\theta$.

Note that for a given destination $\mathbf{x}$ the d-flow intensity $\mathbf{J}(\mathbf{r}, \mathbf{x})$ corresponds to a vector field in $\mathbb{R}^{2}$.

A central concept is the following:

Definition 10 (destination based forwarding (DBF)) In destination based single-path forwarding, the direction $\vartheta$ in which a given packet at $\mathbf{r}$ is forwarded is defined solely by the destination $\mathbf{x}$ of the packet, $\vartheta(\mathbf{r}, \mathbf{x}): \mathbb{R}^{2} \times \mathbb{R}^{2} \rightarrow[0,2 \pi)$.

Consequently, the end-to-end path from $\mathbf{x}_{1}$ to $\mathbf{x}_{2}$, denoted by $p\left(\mathbf{x}_{1}, \mathbf{x}_{2}\right)$, gets defined by the function $\vartheta$, i.e., $\mathcal{P}=\mathcal{P}(\vartheta)$. In $[11,12]$ explicit expressions for calculating the scalar packet flux for arbitrary DBF paths is given. Note that destination based forwarding necessarily implies single-path routes but not vice versa, i.e., $\vartheta$-forwarding rule is a stronger requirement than just a single-path between any given pair of locations, because from $\vartheta$-forwarding rule it follows that all the other nodes on the path to a given destination $\mathbf{x}$ also use the same (remaining) path for the packets, having the destination $\mathrm{x}$. In the present paper, we show that the optimal solution to the load balancing problem can be achieved with destination based forwarding.

We define an additional property that a routing may have:

Definition 11 (bidirectionality) Paths are bidirectional if the paths $p\left(\mathbf{x}_{1}, \mathbf{x}_{2}\right)$ and $p\left(\mathbf{x}_{2}, \mathbf{x}_{1}\right)$ are identical for all $\mathbf{x}_{1}, \mathbf{x}_{2} \in \mathcal{A}$.

Note that a flow on a given path contributes to the scalar flux at any point on the path by an amount equal to the absolute size of the flow, no matter what the direction of the flow is (cf., Def 2). Thus, allowing a different return path is, from the load balancing point of view, in effect equivalent to allowing two paths between each pair of locations.

We denote by $\arg \mathbf{J}(\mathbf{r}, \mathbf{x})$ the angle of direction of the vector $\mathbf{J}(\mathbf{r}, \mathbf{x})$ with respect to an arbitrary (fixed) reference direction. It is straightforward to see that, with single-path DBF routes, we have

$$
\vartheta(\mathbf{r}, \mathbf{x})=\arg \mathbf{J}(\mathbf{r}, \mathbf{x}),
$$

i.e., the paths $p(\mathbf{r}, \mathbf{x})$ follow the field lines of $\mathbf{J}(\mathbf{r}, \mathbf{x})$, and

$$
\varphi(\mathbf{r}, \theta ; \mathbf{x})=\delta(\theta-\vartheta(\mathbf{r}, \mathbf{x})) \cdot|\mathbf{J}(\mathbf{r}, \mathbf{x})| .
$$




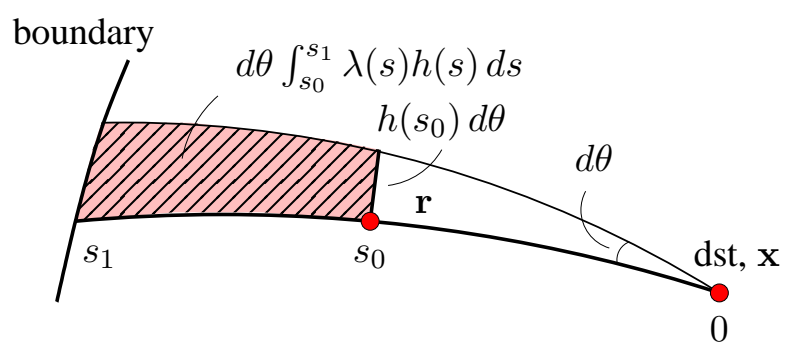

Fig. 2. Expression for d-flow $\mathbf{J}(\mathbf{r}, \mathbf{x})$ with arbitrary single-path routes.

where $\delta(\cdot)$ is Dirac's delta-function. Moreover, for scalar packet flux with destination based single-path routing we have

$$
\Phi(\mathbf{r})=\int_{0}^{2 \pi} \varphi(\mathbf{r}, \theta) d \theta=\int_{0}^{2 \pi} \int_{\mathcal{A}} \varphi(\mathbf{r}, \theta ; \mathbf{x}) d^{2} \mathbf{x} d \theta=\int_{\mathcal{A}}|\mathbf{J}(\mathbf{r}, \mathbf{x})| d^{2} \mathbf{x} .
$$

In general, for an arbitrary domain with destination based single-path routes, there are paths arriving to a given destination $\mathbf{x}$ from all angles $\theta, \theta \in[0,2 \pi)$. Let $\mathbf{p}(s ; \mathbf{x}, \theta)$ denote a point on the path arriving from the direction $\theta$, with the parameter $s$ specifying the distance of $\mathbf{p}$ from $\mathbf{x}$ along the path, i.e., the arc length between $\mathbf{p}$ and $\mathbf{x}$. Here we assume for notational simplicity that there is a one-to-one correspondence between the paths and their direction angles $\theta$ at the destination $\mathbf{x}$. The situation is illustrated in Fig. 2 . (Note that there exist valid though somewhat pathological sets of routes that do not satisfy this assumption. This happens when separate paths leading to $\mathrm{x}$ coalesce at some point, as in the case of so-called radial ring paths discussed later in Section 4.) The magnitude of d-flow at $\mathbf{r}$ to destination $\mathbf{x}$ can be obtained by evaluating the line integral along the path $\mathbf{p}(s ; \mathbf{x}, \theta)$ going through $\mathbf{r}$,

$$
|\mathbf{J}(\mathbf{r}, \mathbf{x})|=\frac{1}{h\left(s_{0}\right)} \int_{s_{0}}^{s_{1}} \lambda(s) h(s) d s
$$

where $\lambda(s)=h(s ; \mathbf{r}, \mathbf{x})$ is the traffic demand density from point $s$ (on path $\mathbf{p}(s ; \mathbf{x}, \theta)$ ) to $\mathbf{x}$, and $h(s)=$ $h(s ; \mathbf{r}, \mathbf{x})$ is divergence rate of paths,

$$
h(s)=\lim _{d \theta \rightarrow 0} \frac{|\mathbf{p}(s ; \mathbf{x}, \theta+d \theta)-\mathbf{p}(s ; \mathbf{x}, \theta)|}{d \theta} .
$$

Thus, $h(s)$ describes the distance between two neighboring paths as illustrated in Fig. 2. For example, this distance is simply a linear function of $s$ for shortest paths, $h(s)=s$. The proof of (9) is straightforward and essentially the same as the proof given in $[11,12]$ for an expression for the scalar packet flux with curvilinear paths.

\section{Main Results}

In this section we prove that the optimal solution to the load balancing problem in the context of dense wireless multi-hop networks can be obtained by using destination based single-path routes. We also give several corollaries related to the optimal solution. 


\subsection{Optimality of field-line routing}

Let $\mathcal{P}$ denote any set of routes and let $\mathbf{J}(\mathbf{r}, \mathbf{x} ; \mathcal{P})$ be the d-flow intensity of the traffic destined to $\mathbf{x}$ using this routing. We associate to $\mathcal{P}$ another routing system $\mathcal{S}(\mathcal{P})$, consisting of destination based single-path routes defined by the forwarding rule

$$
\vartheta(\mathbf{r}, \mathbf{x} ; \mathcal{P})=\arg \mathbf{J}(\mathbf{r}, \mathbf{x} ; \mathcal{P})
$$

That is, the field lines of the d-flow $\mathbf{J}(\mathbf{r}, \mathbf{x} ; \mathcal{P})$ are used as routes for packets with destination $\mathbf{x}$. Accordingly, this associated routing system $\mathcal{S}(\mathcal{P})$ is referred to as the field-line routing. Now we establish our main result. Proposition 12 For any routing system $\mathcal{P}$, the associated field-line routing $\mathcal{S}(\mathcal{P})$ yields a scalar flux that is everywhere less than or equal to that of the original routing $\mathcal{P}$,

$$
\Phi(\mathbf{r} ; \mathcal{S}(\mathcal{P})) \leq \Phi(\mathbf{r} ; \mathcal{P}), \quad \forall \mathbf{r}, \mathcal{P}
$$

Proof: First we show that $\mathbf{J}(\mathbf{r}, \mathbf{x} ; \mathcal{S}(\mathcal{P}))=\mathbf{J}(\mathbf{r}, \mathbf{x} ; \mathcal{P})$. This follows because (i) the fields are by construction everywhere aligned, $\arg \mathbf{J}(\mathbf{r}, \mathbf{x} ; \mathcal{S}(\mathcal{P}))=\arg \mathbf{J}(\mathbf{r}, \mathbf{x} ; \mathcal{P})$, and (ii) they both satisfy the source equation (flow conservation condition)

$$
\nabla \cdot \mathbf{J}(\mathbf{r}, \mathbf{x} ; \mathcal{P})=\nabla \cdot \mathbf{J}(\mathbf{r}, \mathbf{x} ; \mathcal{S}(\mathcal{P}))=\lambda(\mathbf{r}, \mathbf{x})-\delta(\mathbf{r}-\mathbf{x}) \Lambda(\mathbf{x}), \quad \forall \mathbf{r}, \mathbf{x}
$$

where $\Lambda(\mathbf{x})\left[1 / \mathrm{s} / \mathrm{m}^{2}\right]$ denotes the density of total traffic destined to $\mathbf{x}$ (per unit area about $\mathbf{x}$ ), since by construction they both carry the traffic defined by the traffic matrix $\lambda(\mathbf{r}, \mathbf{x})$. Now we need to show that a field with properties (i) and (ii) is unique, which then implies that $\mathbf{J}(\mathbf{r}, \mathbf{x} ; \mathcal{S}(\mathcal{P}))=\mathbf{J}(\mathbf{r}, \mathbf{x} ; \mathcal{P})$. To simplify the notation, we suppress the parameter $\mathrm{x}$ and denote the source density of the right hand side of (12) by $\sigma(\mathbf{r})$. Thus let $\mathbf{J}^{\prime}(\mathbf{r})$ be a solution to $\nabla \cdot \mathbf{J}^{\prime}(\mathbf{r})=\sigma(\mathbf{r})$ that is parallel to a given solution $\mathbf{J}(\mathbf{r})$ everywhere. Now, use a coordinate system defined by the common field lines. Let one of the coordinates be $s$, the curve length parameter along a field line, and let $h(s)$ be the distance of two 'neighboring' field lines at point $s$. By applying Gauss' theorem (see, e.g., [22]) to a small volume element of length $d s$, between the neigboring lines we obtain $\nabla \cdot \mathbf{J}^{\prime}(\mathbf{r})=\frac{1}{h(s)} \frac{d}{d s}\left(h(s)\left|\mathbf{J}^{\prime}(s)\right|\right)$ since $h(s) d s$ is the area of the element and $d(h(s)|\mathbf{J}(s)|)$ is the flow out from one end minus the flow in at the other end. Therefore, the expression represents the net flow out of the element per unit area. Thus, along the field line, $\left|\mathbf{J}^{\prime}(s)\right|$ obeys a first order ordinary differential equation

$$
\frac{d}{d s}\left(h(s)\left|\mathbf{J}^{\prime}(s)\right|\right)=h(s) \sigma(s)
$$

Since at the outer boundary $\mathbf{J}^{\prime}(\mathbf{r})$ is zero, setting a fixed initial condition for the equation, the solution for $\left|\mathbf{J}^{\prime}(\mathbf{r})\right|$ is uniquely defined on any field line. Thus $\mathbf{J}^{\prime}(\mathbf{r})$ has not only the same direction everywhere, but also the same magnitude as $\mathbf{J}(\mathbf{r})$.

Having established that $\mathbf{J}(\mathbf{r}, \mathbf{x} ; \mathcal{S}(\mathcal{P}))=\mathbf{J}(\mathbf{r}, \mathbf{x} ; \mathcal{P})$, we can write by (6)

$$
\int_{0}^{2 \pi} \varphi(\mathbf{r}, \theta ; \mathbf{x}, \mathcal{P}) \mathbf{e}_{\theta} d \theta=\int_{0}^{2 \pi} \varphi(\mathbf{r}, \theta ; \mathbf{x}, \mathcal{S}(\mathcal{P})) \mathbf{e}_{\theta} d \theta
$$


By the triangle inequality we have

$$
\int_{0}^{2 \pi} \varphi(\mathbf{r}, \theta ; \mathbf{x}, \mathcal{P}) d \theta \equiv \int_{0}^{2 \pi}\left|\varphi(\mathbf{r}, \theta ; \mathbf{x}, \mathcal{P}) \mathbf{e}_{\theta}\right| d \theta \geq\left|\int_{0}^{2 \pi} \varphi(\mathbf{r}, \theta ; \mathbf{x}, \mathcal{P}) \mathbf{e}_{\theta} d \theta\right| .
$$

With $S(\mathcal{P})$ the angular d-flux density $\varphi(\mathbf{r}, \theta ; \mathbf{x}, \mathcal{S}(\mathcal{P}))$ is concentrated on a single direction, and the above holds as an equality,

$$
\int_{0}^{2 \pi} \varphi(\mathbf{r}, \theta ; \mathbf{x}, \mathcal{S}(\mathcal{P})) d \theta \equiv \int_{0}^{2 \pi}\left|\varphi(\mathbf{r}, \theta ; \mathbf{x}, \mathcal{S}(\mathcal{P})) \mathbf{e}_{\theta}\right| d \theta=\left|\int_{0}^{2 \pi} \varphi(\mathbf{r}, \theta ; \mathbf{x}, \mathcal{S}(\mathcal{P})) \mathbf{e}_{\theta} d \theta\right|
$$

By (13) the right hand sides are equal, whence

$$
\int_{0}^{2 \pi} \varphi(\mathbf{r}, \theta ; \mathbf{x}, \mathcal{S}(\mathcal{P})) d \theta \leq \int_{0}^{2 \pi} \varphi(\mathbf{r}, \theta ; \mathbf{x}, \mathcal{P}) d \theta
$$

and by integrating over $\mathrm{x}$ the claim (11) follows.

An immediate corollary from Proposition 12 is the following.

Proposition 13 An optimal solution for the load balancing problem (3) can be obtained with a destination based forwarding $(D B F)$.

Proof: Let $\mathcal{P}$ be a routing that realizes the minimum of (3). By Proposition 12, the field-line routing $\mathcal{S}(\mathcal{P})$, which is a destination based single-path routing, has a flux that is nowhere larger than that of $\mathcal{P}$ and thus achieves the optimum.

The proof of Proposition 12 shows, as another corollary, the following local result:

Proposition 14 If a routing system $\mathcal{P}$ at point $\mathbf{r}$ forwards packets with a given destination genuinely to several directions, the corresponding field-line routing $\mathcal{S}(\mathcal{P})$ yields a strictly lower scalar flux about $\mathbf{r}$.

Proof: Under these conditions the triangle inequality relation holds as a strict inequality at $\mathbf{r}$.

Note that forwarding of packets with a common destination to different directions at a given point $\mathbf{r}$ does not imply multi-path routing. Even single-path routing that is not destination based leads to forwarding packets in different directions at an intermediate point, depending on their source points.

As a final remark, we note that, since by Proposition 13 the optimum can be attained by destination based single-path routes, and for those the scalar flux can be written in terms of the d-flow $\mathbf{J}(\mathbf{r}, \mathbf{x})$ as given in (8), one can formulate the load balancing problem solely in terms of $\mathbf{J}(\mathbf{r}, \mathbf{x})$,

$$
\min _{\mathbf{J}(\mathbf{r}, \mathbf{x})} \max _{\mathbf{r}} \int_{\mathcal{A}}|\mathbf{J}(\mathbf{r}, \mathbf{x})| d^{2} \mathbf{x}
$$

subject to the flow-conservation requirement (12),

$$
\nabla \cdot \mathbf{J}(\mathbf{r}, \mathbf{x})=\lambda(\mathbf{r}, \mathbf{x})-\delta(\mathbf{r}-\mathbf{x}) \Lambda(\mathbf{x}), \quad \forall \mathbf{r}, \mathbf{x}
$$

It should be emphasized that the paths do not appear at all in these equations. If the solution $\mathbf{J}(\mathbf{r}, \mathbf{x})$ has been found, the paths can be determined afterwards as the field lines of this d-flow. This formulation, however, will not be pursued further in this paper but is the topic of [23]. 


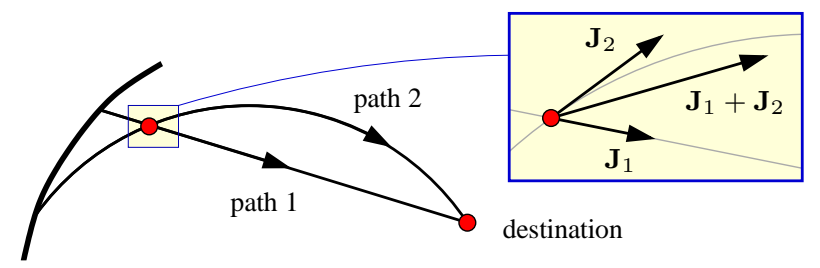

Fig. 3. Transformation from a two-path routing to single-path routing according to (15).

\subsection{Uniqueness of the optimal solution}

Let us next elaborate the main result by characterizing the optimal paths in a so-called bottleneck region. To this end, first consider the following special case of multi-path routing:

Definition 15 (randomized multi-path routing) Let $\mathcal{P}_{i}, i \in I$, be a countable set of single-path solutions to the minmax problem (3), and let $\alpha_{i} \geq 0$ be arbitrary probabilities, $\sum_{i \in I} \alpha_{i}=1$. A routing where a path for each packet is chosen randomly from set I according to the $\alpha_{i}$ is referred to as randomized multi-path routing.

Thus, the path chosen at random by the source for each packet is followed to the destination without any further random selections. It is straightforward to see that,

$$
\Phi(\mathbf{r})=\sum_{i} \alpha_{i} \Phi_{i}(\mathbf{r})
$$

where $\Phi_{i}$ denotes the packet flux when all the traffic is routed using path set $i, i \in I$. Similarly, let the $\mathbf{J}_{i}(\mathbf{r}, \mathbf{x})$ denote the corresponding d-flows when all the traffic destined to $\mathbf{x}$ is routed using path set $i, i \in I$. According to (11), the field-line routing decision is in the direction of the net d-flow, i.e.,

$$
\vartheta(\mathbf{r}, \mathbf{x})=\arg \sum_{i} \alpha_{i} \mathbf{J}_{i}(\mathbf{r}, \mathbf{x})
$$

This is illustrated in Fig. 3 for two alternative path sets. According to Proposition 14, paths defined by (15) lead to a better solution with a lower maximal scalar flux. In Section 4 we illustrate this by means of numerical examples and show that the single-path routing indeed lowers the maximum of the scalar flux.

Typically, an optimal solution for the load balancing problem in finite fixed networks is such that the maximal load is attained in several links. This suggests that the optimal solution in the present context of massively dense networks is also such that the maximal load, denoted by $\Phi_{\text {opt }}$, is obtained in some area $\mathcal{A}^{(B)} \subset \mathcal{A}$. We further believe that $\mathcal{A}^{(B)}$ has a strictly positive area, i.e., it is not a single point.

Definition 16 (bottleneck region) Let I denote the index set of all the optimal solutions to the load balancing problem, i.e., having the same maximal scalar flux $\Phi_{\mathrm{opt}}$. Define the bottleneck region as

$$
\mathcal{A}^{(B)}=\left\{\mathbf{r} \in \mathcal{A}: \Phi_{i}(\mathbf{r})=\Phi_{\mathrm{opt}}, \forall i \in I\right\}
$$

From the optimality it follows that the bottleneck region cannot be empty. To this end, consider an arbitrary finite subset of the optimal single-path solutions $\vartheta_{i}(\mathbf{r}, \mathbf{x})$ yielding the scalar fluxes $\Phi_{i}(\mathbf{r}), i=1, \ldots, n$. The scalar flux for the corresponding multi-path system with random path selection with probabilities $\alpha_{i}$ according to (14) is simply $\Phi(\mathbf{r})=\sum_{i} \alpha_{i} \cdot \Phi_{i}(\mathbf{r})$. In particular, if $\left\{\mathbf{r} \in \mathcal{A}: \Phi_{i}(\mathbf{r})=\Phi_{\text {opt }}, \forall i=1, \ldots, n\right\}=$ 
$\emptyset$, then choosing, e.g., $\alpha_{i}=1 / n$ gives

$$
\max _{\mathbf{x}}\left(\sum_{i} \alpha_{i} \cdot \Phi_{i}(\mathbf{r})\right)<\Phi_{\mathrm{opt}},
$$

which is a contradiction and thus $\mathcal{A}^{(B)}$ is non-empty. So, $\mathcal{A}^{(B)}$ surely contains at least one point. For now, let us assume that the bottleneck region $\mathcal{A}^{(B)}$ is a set with a strictly positive area.

Proposition 17 Optimal paths are unambiguous inside the bottleneck region.

Proof: Let $\vartheta_{1}(\mathbf{r}, \mathbf{x})$ and $\vartheta_{2}(\mathbf{r}, \mathbf{x})$ denote the forwarding functions of two optimal solutions. If

$$
\vartheta_{1}(\mathbf{r}, \mathbf{x}) \neq \vartheta_{2}(\mathbf{r}, \mathbf{x}), \quad \text { for some } \mathbf{r} \in \mathcal{A}^{(B)},
$$

then by Proposition 14 the field-line routing, obtained from the randomized multi-path routing using (15), would give a lower scalar flux at $\mathbf{r}$ contradicting the assumption that $\mathbf{r} \in \mathcal{A}^{(B)}$.

Proposition 18 With strictly positive traffic demands, $\lambda\left(\mathrm{x}_{1}, \mathbf{x}_{2}\right)>0$, the optimal paths are bidirectional in the bottleneck region.

Proof: Consider a point in the bottleneck area, $\mathrm{x}_{1} \in \mathcal{A}^{(B)}$. As the traffic demands are strictly positive there is some traffic flowing from $\mathrm{x}_{1}$ to $\mathrm{x}_{2}$, and vice versa. Let $A$ denote the path from $\mathrm{x}_{1}$ to $\mathrm{x}_{2}$, and $B$ the reverse path. Next consider a multi-path routing where some of the traffic on $A$ has been moved to reverse path $B$, and similarly in the reverse direction. This clearly has no effect on the resulting scalar flux. However, if $A$ is different from $B$ (in the bottleneck region), then this multi-path solution could be improved by using the corresponding field-line routing, which leads to a contradiction.

\section{Numerical Examples}

In this section we illustrate our results in two example geometries: unit disk and unit square, with uniform traffic demands,

$$
\lambda\left(\mathbf{x}_{1}, \mathbf{x}_{2}\right)=\frac{\Lambda}{A^{2}}, \quad \mathbf{x}_{1}, \mathbf{x}_{2} \in \mathcal{A}
$$

where $\Lambda$ denotes the total packet flow [pkt/s] and $A$ denotes the area of $\mathcal{A}$, i.e., $A=\pi$ for the unit disk and $A=1$ for the unit square. In particular, we demonstrate that field-line routing derived from some heuristically defined multi-path routing systems is indeed able to lower the maximum of the scalar flux.

The most obvious choice for paths are the shortest paths (SP), which, in this context, correspond to straight line segments between the source and destination. The local forwarding rule for the shortest paths is simply

$$
\vartheta_{\mathrm{SP}}(\mathbf{r}, \mathbf{x})=\arg (\mathbf{x}-\mathbf{r}) .
$$

Compact expressions for the scalar packet flux $\Phi(\mathbf{r})$ and the d-flow intensity $\mathbf{J}(\mathbf{r}, \mathbf{x})$ exist in this case. Let $a_{1}=a_{1}(\mathbf{r}, \theta)$ denote the distance to the boundary from $\mathbf{r}$ in direction $\theta$, and $a_{2}$ in the opposite direction, $a_{2}(\mathbf{r}, \theta)=a_{1}(\mathbf{r}, \theta+\pi)$. Then it is straightforward to show that the angular flux corresponding to the shortest paths is given by

$$
\varphi_{\mathrm{SP}}(\mathbf{r}, \theta)=\int_{0}^{a_{1}} \int_{0}^{a_{2}} \lambda\left(\mathbf{x}_{1}, \mathbf{x}_{2}\right) \cdot\left(s_{1}+s_{2}\right) d s_{2} d s_{1},
$$

where $\mathbf{x}_{1}=\mathbf{r}-s_{1} \mathbf{e}_{\theta}$ and $\mathbf{x}_{2}=\mathbf{r}-s_{2} \mathbf{e}_{\theta}$ with $\mathbf{e}_{\theta}$ denoting the unit vector in direction $\theta$. Consequently, with 


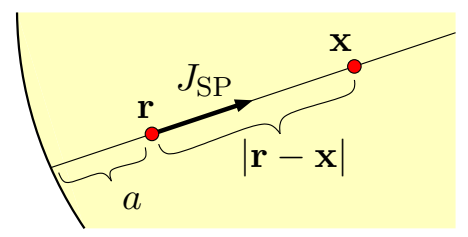

Fig. 4. Notation for the d-flow intensity $J_{\mathrm{SP}}(\mathbf{r}, \mathbf{x})$ with the shortest paths given by (18).

uniform traffic demands, $\lambda\left(\mathbf{x}_{1}, \mathbf{x}_{2}\right)=\Lambda / A^{2}$, the corresponding scalar packet flux is given by

$$
\Phi_{\mathrm{SP}}(\mathbf{r})=\frac{\Lambda}{A^{2}} \int_{0}^{\pi} a_{1} a_{2}\left(a_{1}+a_{2}\right) d \theta .
$$

The d-flow density for shortest paths with uniform traffic demands is given by

$$
\mathbf{J}_{\mathrm{SP}}(\mathbf{r}, \mathbf{x})=\frac{\Lambda}{A^{2}}\left(d+d^{2} / 2\right)(\mathbf{x}-\mathbf{r})
$$

where $d=a /|\mathbf{x}-\mathbf{r}|$ and $a=a(\mathbf{r}, \mathbf{x})$ denotes the distance to the boundary from $\mathbf{r}$ in the direction of $\mathbf{r}-\mathbf{x}$ as illustrated in Fig. 4.

\subsection{Unit Disk}

As a first example we consider the standard example of a circular disk with unit radius (see, e.g., [4, 11, $12,18,19])$. Due to the symmetry, the scalar flux in this system is a function of radius $r$ only.

The scalar flux according to the shortest path routes can be obtained from (17) yielding an elliptic integral of the second kind $[11,12]$,

$$
\Phi_{\mathrm{SP}}(r)=\frac{2\left(1-r^{2}\right) \cdot \Lambda}{\pi^{2}} \int_{0}^{\pi} \sqrt{1-r^{2} \cos ^{2} \phi} d \phi,
$$

which has its maximum at the origin, $\Phi_{\mathrm{SP}}(0)=(2 / \pi) \cdot \Lambda \approx 0.637 \cdot \Lambda$. Applying the lower bounds (4) and (5) for the unit disk with uniform demands gives

$$
\begin{array}{lll}
\Phi_{\mathrm{opt}} \geq \frac{128}{45 \pi^{2}} \cdot \Lambda \approx 0.288 \cdot \Lambda, & & \text { (distance bound) } \\
\Phi_{\mathrm{opt}} \geq \frac{1}{4} \cdot \Lambda=0.25 \cdot \Lambda . & & \text { (cut bound, e.g., along } x \text {-axis) }
\end{array}
$$

\subsubsection{Elementary path sets}

In addition to the shortest paths, some other elementary path sets can be considered in the circular area. In Fig. 5 we have illustrated two single-path forwarding rules that correspond to the so-called inner and outer radial-ring paths [11,12], which are bidirectional and also satisfy the destination based forwarding rule requirement given in Def. 10. These path sets consist of one radial component and one ring component. With the inner radial-ring paths, the order is chosen so that the ring component closer to the origin is used, and for the outer radial-ring paths it is the opposite. Similarly like the shortest paths, these path sets are also not ideal. However, as their simple form facilitates the analysis, they serve as good examples. 

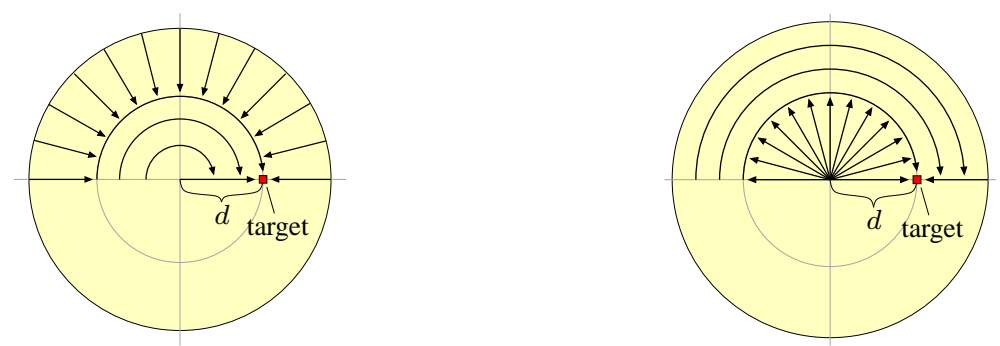

Fig. 5. Local routing decisions $\vartheta(\mathbf{r}, \mathbf{x})$ for inner (left) and outer (right) radial-ring paths for $\mathbf{x}=(d, 0)$ in upper half plane.

Without loss of generality, one can assume that the destination is located on positive $x$-axis, $\mathbf{x}=(d, 0)$. The routing decision for radial-ring paths can be expressed conveniently in polar coordinates. Let $(r, \theta)$ denote the current location $\mathbf{r}$ in the upper half plane. The local routing rules for the inner and outer radialring paths are

$$
\vartheta_{\text {in }}=\left\{\begin{array}{ll}
\theta-\pi, & r>d, \\
\theta-\pi / 2, & r \leq d, \theta>0, \\
0, & r<d, \theta=0,
\end{array} \quad \vartheta_{\text {out }}= \begin{cases}\theta, & r<d, \\
\theta-\pi / 2, & r \geq d, \theta>0, \\
\pi, & r>d, \theta=0 .\end{cases}\right.
$$

The corresponding scalar fluxes are

$$
\Phi_{\text {in }}(r)=\frac{(\pi+1)\left(r-r^{3}\right)}{\pi} \cdot \Lambda, \quad \Phi_{\text {out }}(r)=\frac{(\pi-1) r^{3}+r}{\pi} \cdot \Lambda,
$$

obtaining their maxima $\Phi_{\text {in }} \approx 0.507 \cdot \Lambda$ and $\Phi_{\text {out }}=\Lambda$ at $r=1 / \sqrt{3}$ and $r=1$, respectively.

Note that for radial-ring paths, several paths coalesce before reaching the destination and, thus, packets arriving at a certain angle may belong to different paths. In fact, for any given destination $\mathrm{x}$, packets arrive from one of four possible directions. For the shortest paths, this is not the case and packets arrive from (and depart to) all possible directions.

\subsubsection{Randomized path selection}

In $[11,12]$ it was shown that by using a randomized path selection using two or more single-path routes from a given set of routes, one can achieve a considerably lower maximal scalar packet flux than with any of the single-path routes of the set alone. In particular, two combinations of the shortest paths and the radial-ring paths were considered:

(i) shortest paths and outer radial-ring paths,

(ii) shortest paths and outer and inner radial-ring paths.

The optimized path selection probabilities were such that for the resulting scalar packet flux we have (subscript MP denotes multi-path)

$$
\begin{aligned}
& \Phi_{\mathrm{MP} 1}(r)=0.61 \cdot \Phi_{\mathrm{SP}}(r)+0.39 \cdot \Phi_{\mathrm{out}}(r), \\
& \Phi_{\mathrm{MP} 2}(r)=0.5027 \cdot \Phi_{\mathrm{SP}}(r)+0.3763 \cdot \Phi_{\mathrm{out}}(r)+0.121 \cdot \Phi_{\mathrm{in}}(r),
\end{aligned}
$$

where the former yields a maximum flux of $0.397 \cdot \Lambda$, and the latter a maximum flux of $0.3763 \cdot \Lambda$, i.e., the flux corresponding to the outer radial-ring paths at the boundary. The numerical results are given in Table 1, 
Table 1

Results with the shortest paths, and 2- and 3- multi-path routes together with the respective field-line routes. The columns under Proportions describe the used mixture of path sets for each row, and the columns under Max. packet flux give the corresponding performance for the multi-path system (left) and the field-line routes (right).

\begin{tabular}{l|lll|rr} 
& \multicolumn{3}{|c|}{ Proportions } & \multicolumn{2}{c}{ Max. packet flux $\Phi$} \\
& shortest & outer & inner & multi-path & field-line \\
\hline & 1.00 & & & 0.637 & (same) \\
$1)$ & 0.61 & 0.39 & & 0.397 & 0.390 \\
$2)$ & 0.503 & 0.376 & 0.121 & 0.376 & 0.344 \\
$3)$ & 0.437 & 0.343 & 0.22 & 0.389 & 0.343
\end{tabular}
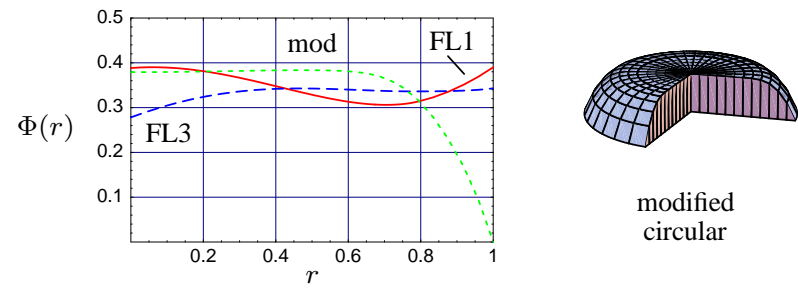

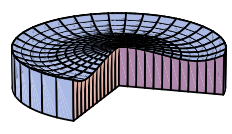

field-line FL1 (2 path sets)

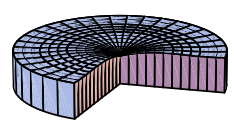

field-line FL3 (3 path sets)

Fig. 6. Resulting scalar flux as a function of distance $r$ from the origin for modified circular paths (see [12]), and the optimized single-path field-line routes FL1 and FL3 (cf., rows 1) and 3) in Table 1). Three dimensional plots illustrate the same situation.

where rows indicated with 1) and 2) correspond to the optimal weights for randomized path selection with the given two and three path sets, respectively, and column "multi-path" contains the corresponding maximal scalar fluxes.

However, according to Proposition 13, multi-path routes MP1 and MP2 cannot be an optimal solution to the load balancing problem and, in particular, the corresponding single-path field-line routes, denoted by FL1 and FL2, obtained using (15) yield a lower maximal scalar flux. This maximal scalar flux can be computed numerically and the corresponding results are given in the column "field-line" in Table 1. We note that in both cases, replacing the multi-path routing with the corresponding single-path field-line routing improves the results considerably.

\subsubsection{Further Optimization}

Instead of using the proportions optimal with respect to the randomized multi-path routing, one can also treat the route selection probabilities $\alpha_{i}$ as free optimization parameters for the resulting single-path routing. As an example, let us consider combinations of the same basic routes consisting of two and three path sets. It turns out that in this case, the optimal proportions for the two path sets (straight and outer) remain the same, as given at row 1) in Table 1. However, for the three path sets (straight, outer and inner), the optimal proportions are different and are given at row 3). In particular with these optimized weights, the corresponding field-line route set, denoted by FL3, yields a maximal scalar packet flux of $0.343 \cdot \Lambda$, which is, to the best of our knowledge, considerably less than which is obtained with any previously proposed set of paths. For reference, the circular paths proposed in [11] yield a maximal flux of $0.424 \cdot \Lambda$, and in [12], a modified version of this path set, after numerical optimization, gives a maximal flux of $0.384 \cdot \Lambda$. Also the so-called curveball routing proposed by Popa et al. [10] is expected to provide a similar performance. Although the idea behind the curveball routes (i.e., defining the paths via a mapping between the plane and a sphere) is appealing, the resulting scalar flux is difficult to analyze exactly.

The resulting scalar fluxes for the single-path routes FL1 and FL3 are illustrated in Fig. 6. Interestingly with FL3, the scalar packet flux at the center of the area is clearly lower than the maximum. This suggests 

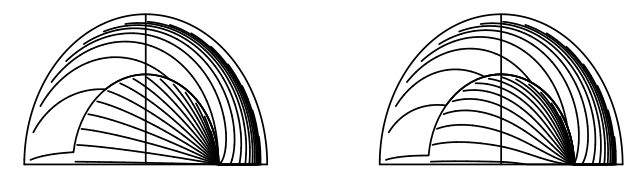

Fig. 7. Single-path routes towards point $(0.6,0)$ obtained by combining multi-path routes MP1 and MP3 to the corresponding field-line routes FL1 and FL3 (rows 1) and 3) in Table 1).
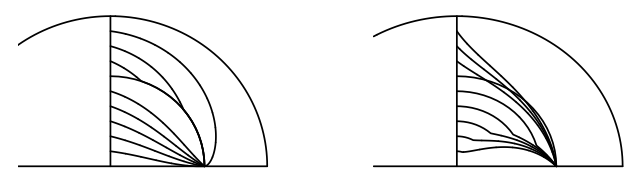

Fig. 8. Single-path routes FL3 from the positive $x$-axis to $(0.6,0)$ (left fig.), and the reverse paths from $(0.6,0)$ back to positive $x$-axis (right fig.). Paths are clearly not bidirectional.

that there is still some room for improvement, e.g., by choosing a different set of base routes.

Fig. 7 illustrates the single-path routes FL1 and FL3 for destination point $\mathbf{x}=(0.6,0)$ (the lower half plane is symmetric). From the figure, it can be seen that the ring with radius 0.6 has a specific role and acts as a "highway" towards the destination. This is due to the fact that both radial-ring paths guide most of the traffic going to $(0.6,0)$ to this ring yielding a singularity in the corresponding d-flow (i.e., a delta function). This singularity is then also present in the resulting single-path routes. Intuitively, from the figure, one can see that neither of these single-path routes can be optimal, e.g., paths just above point $(1-\epsilon, 0)$ are perpendicular to the "correct" direction. The resulting paths deviate from the intuitive ones even more when $d \rightarrow 0$ or when $d \rightarrow 1$.

Finally, we note that the obtained destination based single-path routes are not generally bidirectional. This can be seen, for example, from Fig. 8 which illustrates the single-path routes FL3. The left graph depicts paths from the positive $x$-axis to $(0.6,0)$ and the graph on the right depicts the reverse paths from $(0.6,0)$ back to the positive $x$-axis. Clearly, the paths are different, and thus the single-path routes are not bidirectional. This, as well as the fact that some of the reverse paths cross each other twice, suggests that a set of paths exists which attains even smaller maximal scalar flux. However, we believe that the possible improvement is relatively small and that the FL3 paths are already close to optimal.

\subsection{Unit Square}

Now the considered region $\mathcal{A}$ is a square bounded by the lines $x= \pm 1 / 2$ and $y= \pm 1 / 2$ and having the area $A=1$. The traffic demands are assumed to be uniform, $\lambda(\mathbf{r}, \mathbf{x})=\Lambda / A^{2}=\Lambda$.

We analyze three heuristic path sets. The first set consists of the shortest path routes. The scalar packet flux for these is straightforward to compute using (17) and the maximum is obtained at the origin,

$$
\Phi_{\mathrm{SP}}(0,0)=\frac{\Lambda}{2}(\sqrt{2}+\operatorname{arcsinh} 1) \approx 1.148 \cdot \Lambda .
$$

The mean path length with SP routes is

$$
\bar{\ell}_{\mathrm{SP}}=\frac{1}{15}(2+\sqrt{2}+5 \log (1+\sqrt{2})) \approx 0.521 .
$$

The resulting scalar packet flux distribution is illustrated in Fig. 11. For the two lower bounds (4) and (5) 

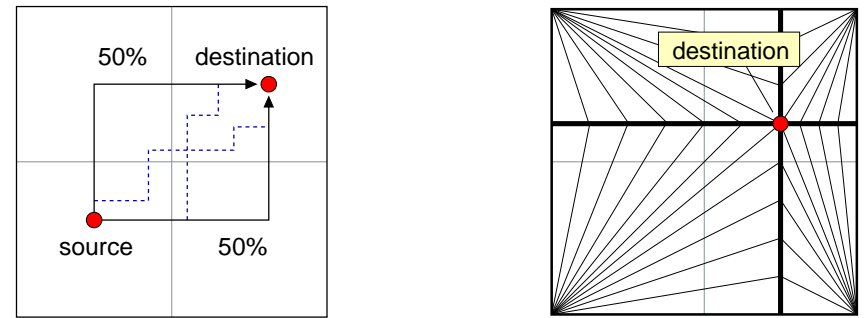

Fig. 9. In the left figure, the Manhattan paths $(\mathrm{MH})$ are illustrated. These paths are based on an imaginary dense Manhattan grid from which one of the outermost shortest path is selected randomly with 0.5 probability. The right figure illustrates the field-line paths resulting from the Manhattan multi-path routes of the left figure.

given in Section 2 we have,

$$
\begin{array}{ll}
\Phi_{\mathrm{opt}} \geq \frac{2 \cdot \Lambda \cdot A_{1} A_{2}}{L A^{2}}=\frac{\Lambda}{2}, & \text { (cut bound along } x \text {-axis) } \\
\Phi_{\mathrm{opt}} \geq \frac{\Lambda \cdot \bar{\ell}_{\mathrm{SP}}}{A} \approx 0.521 \cdot \Lambda . & \text { (distance bound) }
\end{array}
$$

Hence, the distance bound provides a slightly stricter lower bound in this case.

For an arbitrary $h \times 1 / h$ rectangle with unit area the cut bound gives $\Phi_{\text {opt }} \geq \Lambda h / 2$, and the distance bound $\Phi_{\mathrm{opt}} \geq \lambda \cdot \bar{\ell}_{\mathrm{SP}}(h)$, where $\bar{\ell}_{\mathrm{SP}}(h)$ can be obtained numerically. In particular, it turns out that the cut bound gives a better lower bound for all $h \geq 1.0443$ (i.e., the cut bound works well with asymmetric areas).

\subsubsection{Manhattan paths (MH)}

An alternative heuristic path set has its origin in the so-called Manhattan grid. Assume that there is a very dense grid where, at each point in time, one can only move either horizontally or vertically. Clearly, a number of different shortest paths exist between two locations in such a grid except in the special cases when the locations are aligned in the direction of the grid (i.e., either $x$-axis or $y$-axis). In the limit of a dense grid, the number of possible paths tends to infinity. However, in order to avoid the congestion in the center, we use only the two outermost paths with a randomized routing strategy as illustrated in Fig. 9 and refer to these paths as Manhattan paths $(\mathrm{MH})$. For $\mathrm{MH}$ paths, it is straightforward to show that the scalar flux is simply

$$
\Phi_{\mathrm{MH}}(x, y)=\Lambda\left(1-2 x^{2}-2 y^{2}\right),
$$

yielding the maximum at the origin, $\Phi_{\mathrm{MH}}(0,0)=\Lambda$, which corresponds to about $13 \%$ decrease in comparison to the shortest path routes. Furthermore, for any Manhattan type set of paths where packets are allowed to be transmitted only in a horizontal or vertical direction, it is easy to see that the mean path length is $2 / 3$ (or more). Substituting this into the distance bound (4) gives,

$$
\max _{\mathbf{r}} \Phi(\mathbf{r}) \geq \frac{2}{3} \cdot \Lambda, \quad \forall \text { Manhattan type of paths. }
$$

Applying the Manhattan paths to a general $h \times 1 / h$ unit rectangle with uniform traffic demands gives,

$$
\Phi_{\mathrm{MH}}(x, y)=\Lambda\left(\frac{1+h^{2}}{2 h}-2 h y^{2}-\frac{2}{h} x^{2}\right)
$$

i.e., the maximal scalar packet flux is again attained at the origin, $\Phi_{\mathrm{MH}}(0,0)=\Lambda\left(1+h^{2}\right) /(2 h)$. For large 
values of $h$ we have $\Phi_{\mathrm{MH}}(0,0) \rightarrow \Lambda h / 2$, which is equal to the lower bound given by the cut bound (5) showing that the Manhattan paths are asymptotically optimal.

\subsubsection{Field-line routing applied to Manhattan paths}

Manhattan paths as defined above represent a genuine multi-path set of routes where $50 \%$ of the traffic is routed along the "clockwise" path and $50 \%$ along the "anti-clockwise" path (see Fig. 9 left). In order to proceed further, we will next consider the corresponding d-flow intensities, which then allow us to derive the single-path field-line routes having the same or better performance.

For Manhattan paths the d-flow density consists of both a horizontal and vertical component. Let $\mathbf{x}=$ $\left(x_{1}, x_{2}\right)$ and $\mathbf{r}=\left(r_{1}, r_{2}\right)$. Assume first that $r_{1}<x_{1}$ and $r_{2}<x_{2}$ as illustrated in Fig. 10, where $\mathbf{x}$ is an arbitrary interior point of the depicted destination area. Half of the packets with destination $\mathrm{x}$ originating from either strip (lengths $a$ and $b$ ) will be forwarded by a node about $\mathbf{r}$. Moreover, these are the only packets seen at $\mathbf{r}$ and having a destination about $\mathbf{x}$. Thus, for a given $\mathbf{r}$ the d-flow density $\mathbf{J}(\mathbf{r}, \mathbf{x})$ is constant for all $\mathrm{x}$ in the rectangular destination area depicted in the upper right corner and, in particular, the magnitude of $\mathbf{J}(\mathbf{r}, \mathbf{x})$ is directly proportional to the diameter of the rectangular area in the lower left corner, i.e., for $r_{1}<x_{1}$ and $r_{2}<x_{2}$ we have

$$
\mathbf{J}(\mathbf{r}, \mathbf{x})=\frac{\Lambda}{2 A^{2}}(a, b)=\frac{\Lambda}{4}(\hat{\mathbf{i}}+\hat{\mathbf{j}}+2 \mathbf{r}) .
$$

For $r_{1}=x_{1}$ a singularity occurs when half of the packets originating from the area below $r_{2}$ travel via $\mathbf{r}$, and similarly for $r_{2}=x_{2}$, yielding two additional terms,

$$
\Lambda\left(\left(1 / 2+r_{1}\right) \delta\left(x_{2}-r_{2}\right) \hat{\mathbf{i}}+\left(1 / 2+r_{2}\right) \delta\left(x_{1}-r_{1}\right) \hat{\mathbf{j}}\right) .
$$

Combining these together with the other three possible destination areas (note the symmetries) gives a general expression for d-flow density,

$$
\begin{aligned}
\mathbf{J}_{\mathrm{MH}}(\mathbf{r}, \mathbf{x})=\frac{\Lambda}{4}[2 \mathbf{r} & +\operatorname{sgn}\left(r_{1}-x_{1}\right) \hat{\mathbf{i}}+\operatorname{sgn}\left(r_{2}-x_{2}\right) \hat{\mathbf{j}} \\
& +2\left(\operatorname{sgn}\left(x_{1}-r_{1}\right)+2 r_{1}\right) \delta\left(x_{2}-r_{2}\right) \hat{\mathbf{i}} \\
& \left.+2\left(\operatorname{sgn}\left(x_{2}-r_{2}\right)+2 r_{2}\right) \delta\left(x_{1}-r_{1}\right) \hat{\mathbf{j}}\right], \quad \text { for } \mathbf{r} \neq \mathbf{x} .
\end{aligned}
$$

The field-line routes follow when packets with a common destination about $\mathrm{x}$ are forwarded in the direction of $\mathbf{J}_{\mathrm{MH}}(\mathbf{r}, \mathbf{x})$ at every point $\mathbf{r}$. Due to the simple form of $\mathbf{J}_{\mathrm{MH}}(\mathbf{r}, \mathbf{x})$ the field-line routes turn out to be rather elementary as illustrated in Fig. 9 (right) for a single destination $\mathbf{x}$. Note that the horizontal line segment $y=x_{2}$ and vertical line segment $x=x_{1}$ correspond to singularities in $\mathbf{J}_{\mathrm{MH}}(\mathbf{r}, \mathbf{x})$ as all the packets join one of these four main "highways" before reaching destination $\mathbf{x}$.

In the general case, the scalar flux can be computed by evaluating the integral

$$
\Phi(\mathbf{r})=\int_{\mathcal{A}}|\mathbf{J}(\mathbf{r}, \mathbf{x})| d^{2} \mathbf{x} .
$$

Terms corresponding to singularities can be computed separately yielding,

$$
\Phi_{\mathrm{FL}}^{(1)}(\mathbf{r})=\frac{\Lambda}{2}\left(1-2 x^{2}-2 y^{2}\right)=\frac{1}{2} \Phi_{\mathrm{MH}}(\mathbf{r}) .
$$




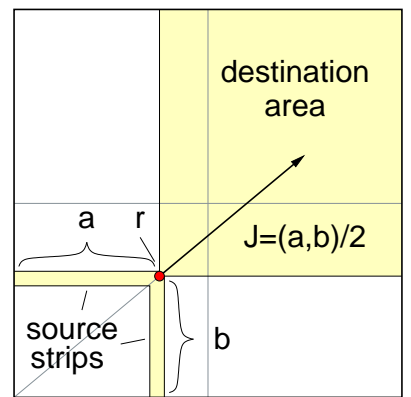

Fig. 10. Derivation of the d-flow densities for Manhattan grid. Note that at every point packets are forwarded only in one of the four possible directions.

For the rest we refer again to Fig. 10 and (19). Consider any destination $\mathrm{x}$ located in the rectangular destination set, which has an area equal to

$$
A_{d}=\left(1 / 2-x_{1}\right)\left(1 / 2-x_{2}\right) .
$$

As mentioned, packets forwarded at $\mathbf{r}$ with such a destination must originate from either strip (lengths $a$ and $b$ ), and, in particular, the d-flow density $\mathbf{J}(\mathbf{r}, \mathbf{x})$ is independent of $\mathbf{x}$,

$$
|\mathbf{J}(\mathbf{r}, \mathbf{x})|=\frac{\Lambda}{2} \sqrt{\left(1 / 2+r_{1}\right)^{2}+\left(1 / 2+r_{2}\right)^{2}} .
$$

Integral of a constant over an area $A_{d}$ trivially gives,

$$
\frac{\Lambda}{8}\left(1-2 x_{1}\right)\left(1-2 x_{2}\right) \sqrt{\left(1+2 r_{1}\right)^{2}+\left(1+2 r_{2}\right)^{2}} .
$$

Taking into account the symmetries then gives,

$$
\begin{aligned}
\Phi_{\mathrm{FL}}^{(2)}(\mathbf{r})=\frac{\Lambda}{16} & {\left[(1-2 x)(1-2 y) \sqrt{(1+2 x)^{2}+(1+2 y)^{2}}\right.} \\
& +(1+2 x)(1-2 y) \sqrt{(1-2 x)^{2}+(1+2 y)^{2}} \\
& +(1-2 x)(1+2 y) \sqrt{(1+2 x)^{2}+(1-2 y)^{2}} \\
& \left.+(1+2 x)(1+2 y) \sqrt{(1-2 x)^{2}+(1-2 y)^{2}}\right] .
\end{aligned}
$$

Combining (20) and (21) then gives a final expression for the scalar packet flux with field-line routing,

$$
\Phi_{\mathrm{FL}}(\mathbf{r})=\Phi_{\mathrm{FL}}^{(1)}(\mathbf{r})+\Phi_{\mathrm{FL}}^{(2)}(\mathbf{r})
$$

With these paths, the scalar packet flux again obtains the maximum at the origin,

$$
\Phi_{\mathrm{FL}}(0,0)=\frac{2+\sqrt{2}}{4} \cdot \Lambda \approx 0.854 \cdot \Lambda,
$$

corresponding to about $26 \%$ improvement in comparison to the shortest paths. The scalar flux distributions corresponding to these three path sets are illustrated in Fig. 11 and Fig. 12. One can observe that some 


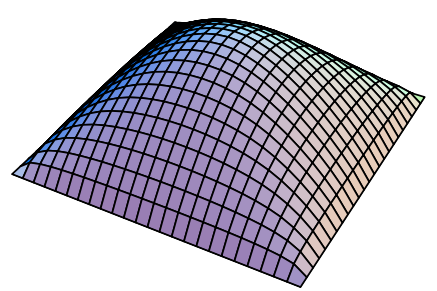

(i) Shortest (SP)

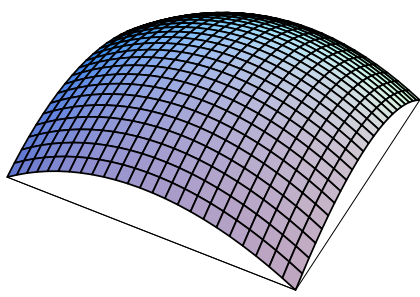

(ii) Manhattan (MH)

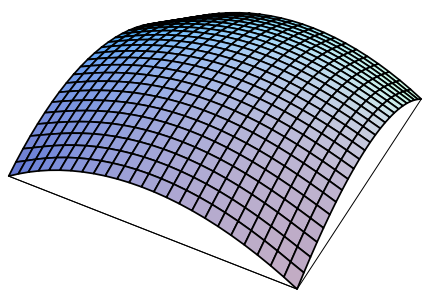

(iii) Field-line (FL)

Fig. 11. Scalar flux distribution for different path sets in unit square in the order of the performance. The field-line routes are obtained from Manhattan paths.

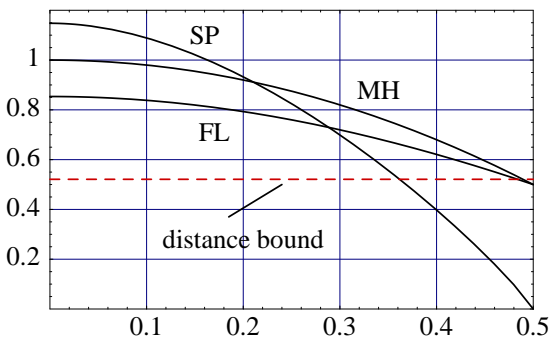

(i) Along $x$-axis (or $y$-axis)

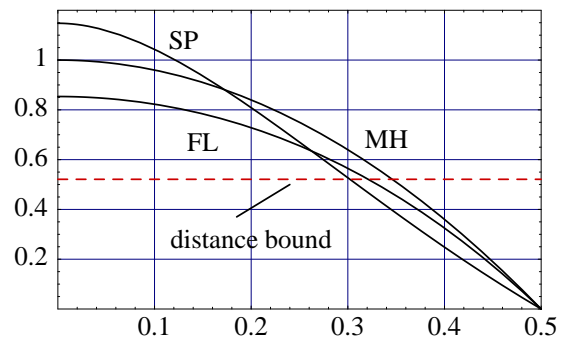

(ii) Along a diagonal

Fig. 12. Resulting scalar fluxes in unit square for shortest paths (SP), Manhattan paths (MH) and field-line routes when applied to the Manhattan routes (FL).

unused capacity exists in each case, especially near the corners. However, taking into account the simple form of the paths (see Fig. 9 right), the results are surprisingly good, and, in particular, the transformation of the multi-path Manhattan routes to single-path field-line routes also led to a considerable improvement in this case.

\subsection{Discussion}

The shortest paths yield, by definition, the shortest possible mean path length, or equivalently, the smallest possible average scalar packet flux. In order to lower the maximal scalar packet flux, one needs to bend some paths away from the congested area, which increases the mean path length and, thus, also the average scalar flux. In other words, there is a trade-off between the achievable minimum maximal scalar flux and the average scalar flux, i.e., the distance bound (4),

$$
\max _{\mathbf{r}} \Phi_{\mathcal{P}}(\mathbf{r}) \geq \frac{\Lambda \cdot \bar{\ell}(\mathcal{P})}{A}
$$

where $\bar{\ell}(\mathcal{P})$ denotes the mean path length with path set $\mathcal{P}$. Additionally, the cut bound (5) provides another lower bound. The performance of different path sets and the lower bounds are illustrated in Fig. 13. In particular, for unit disk we observe that the new solution FL3 is very close to the lower bound (22). In fact, all path sets located on the distance bound (22) have flat scalar packet flux distributions.

For the unit square, the areas near the corners seem to be hard to utilize (i.e., there will be excess capacity in those areas). In particular, we anticipate that the bottleneck region in this case includes the two line segments along the $x$ - and $y$-axis. We also note that the transformation of the heuristic Manhattan path 


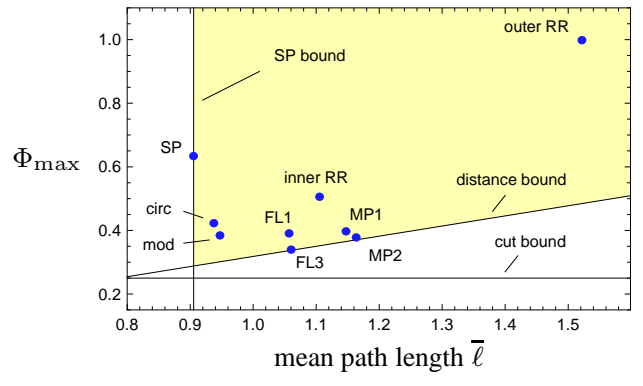

a) unit disk

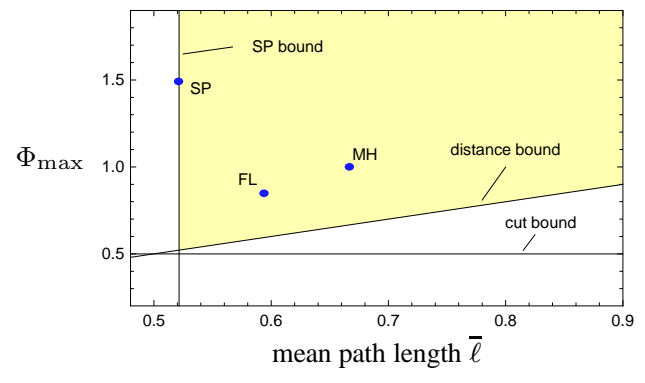

b) unit square

Fig. 13. Trade-off between the maximal scalar flux ( $y$-axis) and the mean path length ( $x$-axis) for different path sets together with two lower bounds (see $[11,12,20]$ ).

set $(\mathrm{MH})$ to the corresponding field-line routing (FL) has improved both the maximal packet flux and the mean path length considerably. However, both path sets are still quite far from the lower bounds, suggesting that even better path sets exist (see, e.g., [23]).

\section{Conclusions}

In this paper, we have studied the load balancing problem in massively dense (wireless) multi-hop networks. The task is to find a set of routes, the minimizing the maximal nodal forwarding load in the network, represented by the so-called scalar flux. In particular, we have focused on comparing multi-path routing with single-path routing. The main contribution of this paper was to show that optimal load balancing can always be obtained by a properly chosen field-line routing, which belongs to the category of destination based single-path routings. This is in striking contrast with the traditional fixed (finite) networks, where the use of multiple paths often yields a better load balancing and higher throughput in the network. We further anticipate that the optimal paths for large but finite networks are similar, while occasionally multiple paths are needed in order to reach the optimum. Moreover, we have shown that, in the bottleneck area, the optimal paths are unique and bidirectional, meaning that the same path is traversed in both directions.

The framework and new results were illustrated by means of numerical examples in elementary domains with uniform traffic demands. A clear gain was achieved by converting multi-path routes in a systematic way into single-path routes. The new routes obtained for a multi-hop network in a unit disk outperform the single- and multi-path solutions given in $[11,12]$. In particular, the best optimized single-path solution (FL3) yields a maximal packet flux of $0.343 \cdot \Lambda$ corresponding to about $46 \%$ improvement in comparison with the shortest path routes, which tend to unnecessarily concentrate too much traffic in the center of the network. Similarly, for the unit square, two elementary sets of paths were first analyzed (shortest paths and Manhattan paths), and then the multi-path Manhattan routes were transformed to the corresponding single-path field-line routes, yielding a significant improvement.

\section{Acknowledgements}

For the part of E. Hyytiä, this work has been performed at the Telecommunications Research Center Vienna (ftw.), which is supported by the Austrian Government and the City of Vienna within the competence center program COMET. For the part of J. Virtamo, this work was performed in the project Fancy funded by the Academy of Finland (grant n:o 210275). 


\section{References}

[1] D. Bertsekas, R. Gallager, Data Networks, 2nd Edition, Prentice-Hall, 1992.

[2] S. Toumpis, L. Tassiulas, Optimal deployment of large wireless sensor networks, IEEE Trans. Information Theory 52 (7) (2006) $2935-2953$.

[3] M. Kalantari, M. Shayman, Routing in wireless ad hoc networks by analogy to electrostatic theory, in: Proceedings of IEEE International Conference on Communications (ICC'04), Paris, France, 2004, pp. 4028-4033.

[4] P. Jacquet, Geometry of information propagation in massively dense ad hoc networks, in: Proceedings of ACM MobiHoc, Roppongi Hills, Japan, 2004, pp. 157-162.

[5] S. Toumpis, Optimal design and operation of massively dense wireless networks, in: Proceedings of ACM Inter-Perf'06, 2006.

[6] R. Catanuto, G. Morabito, Optimal routing in dense wireless multihop networks as a geometrical optics solution to the problem of variations, in: Proceedings of IEEE International Conference on Communications (ICC'06), Istanbul, Turkey, 2006, pp. $134-139$.

[7] R. Catanuto, S. Toumpis, G. Morabito, Opti $\{\mathrm{c}, \mathrm{m}\}$ al: Optical/optimal routing in massively dense wireless networks, in: Proceedings of IEEE Infocom '07, Anchorage, Alaska, 2007, pp. 1010-1018.

[8] N. Shroff, S. Kwon, Paradox of shortest path routing for large multi-hop wireless networks, in: Proceedings of IEEE Infocom '07, Anchorage, Alaska, 2007, pp. 1001-1009.

[9] S. Toumpis, Mother nature knows best: A survey of recent results on wireless networks based on analogies with physics, Computer Networks 58 (2008) 360-383.

[10] L. Popa, A. Rostami, R. M. Karp, C. Papadimitriou, I. Stoica, Balancing the traffic load in wireless networks with curveball routing, in: Proceedings of the 8th ACM International Symposium on Mobile Ad Hoc Networking and Computing (MobiHoc), Montréal, Canada, 2007, pp. 170-179.

[11] E. Hyytiä, J. Virtamo, On load balancing in a dense wireless multihop network, in: Proceedings of NGI 2006, 2nd Conference on Next Generation Internet Design and Engineering, IEEE, València, Spain, 2006, pp. 72-79.

[12] E. Hyytiä, J. Virtamo, On traffic load distribution and load balancing in dense wireless multihop networks, EURASIP Journal on Wireless Communications and Networking, May 2007, Special Issue on Novel Techniques for Analysis \& Design of Cross-Layer Optimized Wireless Sensor Networks.

[13] D. Niculescu, B. Nath, Trajectory based forwarding and its applications, in: Proceedings of the 9th annual international conference on Mobile computing and networking (MobiCom), ACM, San Diego, CA, USA, 2003, pp. 260-272.

[14] F. Kuhn, R. Wattenhofer, A. Zollinger, Asymptotically Optimal Geometric Mobile Ad-Hoc Routing, in: Proceedings of 6th International Workshop on Discrete Algorithms and Methods for Mobile Computing and Communications (DIALM), Atlanta, Georgia, 2002 , pp. $24-33$.

[15] F. Kuhn, R. Wattenhofer, Y. Zhang, A. Zollinger, Geometric ad-hoc routing: Of theory and practice, in: Proceedings of ACM PODC'03, 2003 , pp. 63-72.

[16] H. Frey, Scalable geographic routing algorithms for wireless ad hoc networks, IEEE Network Magazine 18 (4) (2004) 18-22.

[17] R. Baumann, S. Heimlicher, B. Plattner, Routing in large-scale wireless mesh networks using temperature fields, IEEE Network 22 (1) (2008) 25-31.

[18] P. P. Pham, S. Perreau, Performance analysis of reactive shortest path and multi-path routing mechanism with load balance, in: Proceedings of IEEE Infocom '03, Vol. 1, San Francisco, USA, 2003, pp. 251-259.

[19] Y. Ganjali, A. Keshavarzian, Load balancing in ad hoc networks: Single-path routing vs. multi-path routing, in: Proceedings of IEEE Infocom '04, Hong Kong, 2004, pp. 1120-1125.

[20] E. Hyytiä, J. Virtamo, On optimality of single-path routes in massively dense wireless multi-hop networks, in: Proceedings of the 10th ACM International Symposium on Modeling, Analysis and Simulation of Wireless and Mobile Systems (MSWiM), Crete Island, Greece, 2007, pp. 28-35.

[21] G. Bell, S. Glasstone, Nuclear Reactor Theory, Reinhold, 1970.

[22] G. B. Arfken, H. J. Weber, Mathematical Methods for Physicists, sixth Edition, Elsevier Academic Press, 2005.

[23] E. Hyytiä, J. Virtamo, Near-optimal load balancing in dense wireless multi-hop networks, in: Proceedings of NGI 2008, 4th Conference on Next Generation Internet Networks, IEEE, Kraków, Poland, 2008, pp. 181-188.

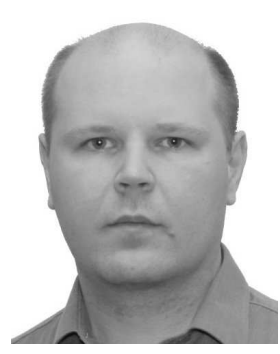

Esa Hyytiä received the M.Sc. (Tech.) degree in engineering physics and Lic.Sc. (Tech.) and Dr.Sc. (Tech.) degrees in electrical engineering from the Helsinki University of Technology, in 1998, 2001 and 2004, respectively. In 2005 he joined the Centre for Quantifiable Quality of Service in Communication Systems (Q2S) at the Norwegian University of Science and Technology. Since 2006 he has been a senior researcher at the Telecommunications Research Center Vienna (ftw.). His research interests include performance analysis, design and optimization of wireless networks, and characterization and analysis of Internet traffic.

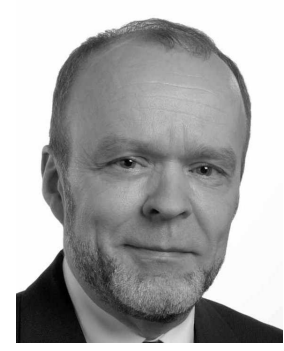

Jorma Virtamo received the M.Sc. (Tech.) degree in engineering physics and D.Sc. (Tech.) degree in theoretical physics from Helsinki University of Technology, in 1970 and 1976, respectively. In 1986, he joined Technical Research Centre of Finland, VTT Information Technology, where he led the teletraffic research group, and became a Research Professor in 1995. Since 1997 he has been a Professor in the Department of Communications and Networking of Helsinki University of Technology. His current research interests include queueing theory and performance analysis of the Internet, multihop wireless networks and peer-to-peer networks. 